Results Findings are presented in 5 acts that demonstrate how the researcher was able to engage with the community and allow findings to be applied in real time. In addition to developing a process model of service delivery (Act 1), the researcher: supported the development and implementation of an integrated reproductive health program (Act 2); tracked, documented, and evaluated a knowledge transfer intervention on maternal opioid use (Act 3), and helped design a countywide demonstration project to improve service utilization for the population (Act 4). The fifth act outlines future directions for a full-scale dissemination study.

Conclusions The community-engaged approach shaped the study's emerging design, which then influenced subsequent program development and delivery. Qualitative methodologies, when combined with community-engaged approaches, can support the timely application of contextualized findings that are delivered through established relationships, using persuasive formats.

\section{P8 THE FUTURE OF SOCIAL RESEARCH ABOUT PAEDIATRIC CARDIOLOGY SERVICES IN THE UK}

Rosa Mendizabal, Priscilla Alderson, Katy Sutcliffe. UCL, London, UK

10.1136/bmjopen-2019-QHRN.43

Before the NHS was established, without state funding few families could afford paediatric services. But since 1948, the NHS has funded and gradually developed the necessary paediatric medical, surgical, nursing and technical services, the training and research. From the 1970s onwards, paediatric cardiology, the care of children born with abnormal hearts, developed exponentially. Research and development have continued. Around 30 abnormalities were identified in the 1970s; today, 3000 innate heart abnormalities are treated by 2500 different techniques. Technologies have transformed the way heart problems are investigated, diagnosed, treated and prevented. The NHS as a national service has also been vital in providing a few highly specialised centres that serve networks of local hospitals, and transfer children swiftly and safely between them.

There are doubts whether the national funding, planning and administration of the NHS will continue, or be broken into local services, overwhelmed by public health and social care needs. Costly super-specialties may be neglected. BREXIT, if it occurs, is likely to increase difficulties with the supply of funding, staffing, equipment, research and medicines.

Social research about the future of paediatric cardiology will need to address: space (relations between local and supraregional centres and international supply chains of staff, research and technologies); time (how past, present and future changes emerge through dynamic processes); the dialectic of structure and agency; uncertainty (and theories of possibility and absence); and inter-disciplinarity that examines and connects health care expertise, planning and administration, staff training and support, policy and funding, and the interpretive views and actual experiences of the involved families and practitioners.

The aim of this paper is to summarise how critical realism offers concepts and analytical frameworks, which connect disparate but essential parts of the complex service, to assist research about the present and future of British paediatric cardiology services.

\section{P9 HOW DO HEALTHCARE PROFESSIONAL PERCEIVE ONLINE INFORMATION AND SUPPORT FOR YOUNG PEOPLE WITH CANCER}

${ }^{1}$ Sarah Lea, ${ }^{1}$ Ana Martins, ${ }^{2}$ Sue Morgan OBE, ${ }^{3} J a m i e$ Cargill, ${ }^{1}$ Lorna Fern. 'University College London Hospitals NHS Foundation Trust, London, UK; ${ }^{2}$ Leeds Teaching Hospitals NHS Foundation Trust, Leeds, UK; ${ }^{3}$ University Hospitals Bristol NHS Foundation Trust, Bristol, UK

\subsection{6/bmjopen-2019-QHRN.44}

Background The internet is integral to young people (YP) providing round-the-clock access to information and support. We previously identified how, when, and why YP use online resources and highlighted variation in how these are introduced by their healthcare team.

Aims We sought to understand how healthcare professionals (HCP) perceived online information and support for young people with cancer.

Methods Semi-structured interviews with 8 HCPs across the UK informed the development of a survey completed by 38 HCPs. Framework analysis was used to identify key themes and the survey was analysed descriptively.

Results Seven themes emerged:

- Views about YPs use of online resources: HCP felt it was important to facilitate access to the internet and it was part of their role to do this safely.

- How YPs online needs change along their cancer timeline: Professionals described increasing internet use nearing the end and after treatment.

- Different platforms where HCPs refer YP online: websites HCPs trusted so recommended to YP.

- Are young people online needs currently met? HCP felt the internet supported factual information about diagnosis and treatment, but most participants agreed unmet needs around other parts of the cancer timeline existed i.e. end-oftreatment.

- Recognition of the emotional relationship between young people and the internet: HCPs described emotions which trigger YP to search i.e. 'panic' and 'curiosity'.

- Barriers and concerns when referring YP to online resources: the biggest perceived barrier to access was costs to the NHS and most concerning factor was keeping YP safe online.

- Strategies used in practice: included the use of caveats, guiding and pre-empting by searching themselves.

Conclusion We will present key findings from each theme and detail recommendations from the study that could be adopted internationally as a benchmark for best practice for online information and support.

\section{P10 END OF LIFE CONVERSATIONS IN THE CRITICAL CARE UNIT}

1,2Douglas Blackwood, 'Jo Arumagam, ${ }^{2}$ Cecilia Vindrola-Padros. 'University College Hospitals London, London, UK; 'university College London, London, UK

\subsection{6/bmjopen-2019-QHRN.45}

Background Several investigations have highlighted variable and sometimes inadequate care at end of life. ${ }^{1,2}$ A consistent theme of guidance and reviews ${ }^{3,4}$ is the importance of good quality communication. Current guidance ${ }^{5}$ does not address the unique circumstances of critical care. Differentiating between treatments which prolong death or enable recovery is 